\title{
Autobiographie d'un pécheur habitué. Sur Marcel Jouhandeau
}

\section{Ornella Tajani}

\section{(C) OpenEdition}

\section{Journals}

\section{Édition électronique}

URL : http://journals.openedition.org/rief/1191

DOI : 10.4000/rief.1191

ISSN : 2240-7456

\section{Éditeur}

Seminario di filologia francese

\section{Référence électronique}

Ornella Tajani, «Autobiographie d'un pécheur habitué. Sur Marcel Jouhandeau », Revue italienne d'études françaises [En ligne], 6 | 2016, mis en ligne le 15 décembre 2016, consulté le 01 mai 2019 URL : http://journals.openedition.org/rief/1191 ; DOI : 10.4000/rief.1191

Ce document a été généré automatiquement le 1 mai 2019.

\section{(c) (i) (9)}

Les contenus de la RIEF sont mis à disposition selon les termes de la Licence Creative Commons Attribution - Pas d'Utilisation Commerciale - Pas de Modification 4.0 International. 


\title{
Autobiographie d'un pécheur habitué. Sur Marcel Jouhandeau
}

\author{
Ornella Tajani
}

Votre mysticisme me terrifie. [Lettre d'André Gide à Jouhandeau ${ }^{1}$

\section{De l'Enfer au Purgatoire}

1 Le Ciel et l'Enfer : on ne saurait trouver coordonnées plus précises pour définir le cadre existentiel de Marcel Jouhandeau. Cette dichotomie est souvent utilisée par les auteurs qui se sont intéressés au créateur de Chaminadour. Pourtant, entre l'empyrée des gloires littéraires et l'Hadès des écrivains indignes d'être remémorés, c'est plutôt dans le purgatoire de l'oubli que demeure aujourd'hui l'œuvre foisonnante de Jouhandeau : un "saint à rebours ", un « alchimiste du mal » ${ }^{2}$, un « Dr Faust clérical à la recherche de son ipséité éternelle ${ }^{3}$, sont quelques-unes des formules baroques employées pour décrire cet auteur qui a traversé le $\mathrm{XX}^{\mathrm{e}}$ siècle en ayant l'air de courir vers la damnation.

Les études critiques, d'ailleurs peu nombreuses, qui lui sont consacrées, ne manquent jamais d'avancer des explications possibles à cet oubli partiel auquel semble condamnée l'œuvre jouhandélienne : on peut en indiquer d'ordre éditorial, thématique, stylistique et biographique. Je les évoquerai rapidement pour introduire le raisonnement qui nous intéresse.

3 La production de Jouhandeau est abondante : environ cent-vingt titres publiés créent un univers littéraire dans lequel il est difficile de s'y retrouver, tant les œuvres sont de genres variés (contes, nouvelles, anecdotes) ou hybrides (écritures fragmentaires, essais autobiographiques). Certains personnages apparaissent à plusieurs reprises; ainsi, à première vue, il est difficile de comprendre si l'on peut identifier des cycles narratifs à l'intérieur de son œuvre, et si oui, lesquels : sous l'Occupation, par exemple, Jouhandeau dressait lui-même un plan de son œuvre ${ }^{4}$, qui semblait alors être définitif et qui sera pourtant rectifié ensuite. La confusion augmente dès lors que l'on comprend que les 
personnages envisageables comme des sortes d'alter-ego de l'auteur sont au nombre de trois, et assez différents les uns des autres: Théophile, l'amant de Dieu; Monsieur Godeau, le mémorialiste du vice; et Juste Binche, la personnification de la pureté, l'homme « représentant ce que nous pouvons nous permettre de laisser voir de nous à notre père et à notre mère $»^{5}$. C'est là la Sainte Trinité jouhandélienne, ou bien, comme le dit l'auteur lui-même « c'est moi en trois personnes $»^{6}$.

Ses thèmes favoris (la vie de province, les scrupules moraux et religieux liés à son homosexualité, les douleurs du mariage) apparaissent aujourd'hui poussiéreux. Au centre de l'univers littéraire complexe créé par l'auteur, sa relation privilégiée avec le sentiment de l'éternel, qui revêt plusieurs formes. Sa phénoménologie de la province de Chaminadour trouve sa source principale dans sa ville natale, Guéret, dans le Limousin. Chaminadour est un domaine de rêve, aux contours flous, composé par touches comme une fresque, et enveloppé dans un climat d'éternité. «Je suis dans le temps et la flèche qui me transperce est dans l'éternité ${ }^{7}$, écrit Jouhandeau; ou encore : "L'éternel est une dimension indispensable à toute vie intérieure $»^{8}$. La dimension atemporelle de Chaminadour souffre parfois d'inactualité: aujourd'hui, cette province peinte par Jouhandeau, grise, petite, blafarde, n'apparaît pas assez lointaine pour être perçue comme charmante ou exotique, ni assez proche pour toucher réellement le lecteur.

5 L'homosexualité a souvent été définie comme «l'aventure capitale de sa vie »'. Il s'agit toutefois d'une aventure tourmentée de scrupules moraux et souvent marquée par un élan mystique très, voire trop personnel. Voyons un extrait de son essai De l'Abjection :

Un homme qui aime une femme, même s'il l'aime trop, l'aime sans danger absolu, parce qu'il obéit à une loi de sa nature et parce qu'il n'aime en elle que ce qui lui manque à lui, mais un homme qui aime un homme n'aime que l'Homme et il est perdu, parce que c'est sa propre nature qu'il préfère à la Nature entière et que, méprisant le reste de la nature à l'avantage de la sienne, non seulement il se préfère à l'œuvre de Dieu, telle que Dieu l'a faite : il se préfère à Dieu, il préfère sa nature proprement humaine à la nature divine. ${ }^{10}$

Ce fragment montre déjà un aperçu du mouvement de la conscience de Jouhandeau, qui le conduira à se forger une morale à mi-chemin entre l'éthique et l'esthétique, sur laquelle je reviendrai. Certes l'auteur parviendra par la suite à vivre son homosexualité d'une façon moins coupable, il n'en reste pas moins vrai que, comme l'écrira Sartre en 1970 à propos de La Possession: "Jouhandeau est écrasé par l'Église. On ne "liquide" pas le catholicisme : s'il arrive qu'on s'en arrache, c'est à demi-mort et marqué pour toujours $»^{11}$

7 Après la vie de province et l'homosexualité, le troisième volet de la thématique jouhandélienne est le cadre domestique du mariage avec Élise, que l'auteur dépeint volontiers comme une femme affreuse, sorte de Xanthippe mondaine l'obligeant à vivre dans la misère. Jouhandeau entretient avec elle une relation profondément ambiguë, où l'amour, la haine, le mépris, l'indifférence se fondent dans un sentiment insaisissable. Et le désir homosexuel surgit et ressurgit au beau milieu de cette relation comme un spectre, prenant parfois l'apparence du Diable : un Diable de papier, dont la présence littéraire au $\mathrm{XX}^{\mathrm{e}}$ siècle se fait de plus en plus rare, comme l'explique Robert Muchembled dans son étude consacrée à la représentation de Satan en littérature ${ }^{12}$.

8 La langue employée par l'auteur pour raconter ce monde est souvent peu transparente. Son style, brûlant de lyrisme, est « un mélange de profane et de sacré, de baroque et de byzantin $»^{13}$. Son lexique est riche en termes bibliques ou issus du latin et du grec; 
Jouhandeau a une phrase classique, "tendue à l'extrême et néanmoins baroque, au sens où Ponge dit du classicisme qu'il est la corde la plus tendue du baroque $»^{14}$. Sa syntaxe très recherchée fera dire à Claude Mauriac : «La signification réelle de beaucoup de ses phrases nous échappe. [...] Il ne faut pas se laisser distraire. [...] Jouhandeau exige de nous une attention soutenue, mieux, dans le sens bergsonien du mot, une collaboration de tous les instants $»^{15}$.

Enfin, pour ce qui concerne les explications « biographiques » de l'oubli de l'auteur, on ne saurait passer sous silence la question de son antisémitisme, rendu manifeste par la publication de son honteux pamphlet en 1938. Bien qu'il s'agisse d'un antisémitisme circonstanciel et renié par la suite, cela demeure une ombre difficile à oublier ${ }^{16}$.

Malgré tout, en France, et cela jusqu'à sa mort en 1979, Jouhandeau n'a cessé de susciter un certain intérêt : en 1971 encore, les éditions Gallimard publiaient un recueil épistolaire de six cents pages, entièrement constitué des lettres que sa mère lui avait envoyées sa vie durant. Il demeurait cette gloire littéraire de La Nouvelle Revue Française, dont plusieurs œuvres avaient été jugées par André Gide comme "extraordinaires». Par la suite Jouhandeau fut peu ou prou oublié, et cela jusqu'aux années 2000, à partir desquelles il redevint pour quelques auteurs un centre d'intérêt. Les ouvrages le concernant restent toutefois peu nombreux; en France ce sont les mêmes titres qui sont régulièrement réédités et il reste peu traduit à l'étranger : ses trois traductions italiennes ${ }^{17}$ sont passées quasiment inaperçues.

11 Il a souvent été souligné que Jouhandeau faisait partie de ces auteurs qui parviennent à créer " un monde » : il est en effet difficile d'entrer dans ce monde et de le comprendre réellement si l'on se limite à deux ou trois de ses œuvres. Comme l'écrit Jean Gaulmier, l'œuvre jouhandélienne " est comme un miroir brisé dont chaque fragment accroche un reflet du réel. Mais il faut savoir manier le kaléidoscope pour y retrouver la totalité harmonieuse de l'image $»^{18}$. Cette œuvre possède une cohérence interne qui tient lieu de socle pour cet univers lequel, selon les mots de l'auteur, « a plusieurs centres, plusieurs climats, [...] avec des végétations de pensées ${ }^{19}$ très différentes les unes des autres; en revanche, le mouvement interne de ses textes avance dans une seule direction, à l'aune de laquelle Jouhandeau organise et développe son travail. Quelle est donc cette direction?

Au-delà des trois axes thématiques auxquels je faisais allusion (province, homosexualité, mariage), qui divisent son œuvre d'une manière trop tranchée, on peut tenter d'envisager sa production sous un point de vue global : celui de la recherche de la vérité, qui trace un chemin parallèle au parcours biographique de l'auteur. Chaque titre, chaque volume publié est une étape de cette quête incessante de la vérité qui est également, nous le verrons, un chemin de libération. L'œuvre entière de Jouhandeau devient alors une sorte de grande autobiographie, avouée ou déguisée, d'un être qui réunit en lui l'universel et l'extrême singulier. C'est donc sous ce jour qu'il faut la relire aujourd'hui, pour mesurer la véritable ampleur thématique de l'auteur et contempler la constellation que représente ce système littéraire, bâti autour d'une passionnante recherche sur la condition humaine.

13 J'essaierai de montrer comment s'articule cette quête de la vérité, qui constitue au fond la véritable source d'intérêt de la production de Jouhandeau.

14 Les années 1930 sont importantes dans la production de Jouhandeau : c'est au cours de cette décennie qu'il écrit certaines de ses œuvres les plus célèbres, à savoir les recueils Chaminadour I, II et les Chroniques maritales, ainsi que deux de ses essais parmi les plus 
intéressants, Algèbres des valeurs morales et De l'Abjection; enfin, c'est après les années 1930 qu'il commence à parler de son homosexualité de façon explicite ${ }^{20}$. En revanche, c'est en m'appuyant sur des références à deux œuvres moins connues, Ximénès Malinjoude (1927) et L'Amateur d'imprudence (1932), que j'étaierai mon propos. Puis, sur la base des éléments thématiques et stylistiques révélés par l'analyse, je proposerai une interprétation possible des causes de l'oubli de l'auteur.

\section{Une autobiographie permanente}

\section{Ximénès Malinjoude, ou De la religion du Mal}

Ximénès Malinjoude est un récit écrit en 1924 et paru en 1927. Sorte de court roman, il est dédié à Michel Leiris, qui s'est d'ailleurs inspiré de son protagoniste éponyme pour la création d'un de ses personnages, celui de Damoclès dans Aurora; l'inspiration s'exerça d'ailleurs à double sens, car Jouhandeau avait prêté à Ximénès quelques traits de Leiris, à la faveur d'une courte relation amoureuse advenue entre les deux écrivains ${ }^{21}$.

Ce récit diabolique, riche en descriptions surréalistes, où règne une harmonie « magique et homicide $»^{22}$, est l'œuvre dans laquelle l'auteur disait avoir "déposé toute [s]a méchanceté » et où, selon Henri Rode, Jouhandeau tend à créer une région absolue, la région du $\mathrm{mal}^{23}$. Malgré cette singularité, l'ouverture du récit évoque d'emblée la province chère à l'auteur :

Les Malinjoude tenaient une pâtisserie et un dépôt de liqueurs depuis plus d'un siècle dans la Grand'rue. Leur spécialité de crème aux amandes et d'absinthe miellée leur avait créé une réputation qui couvrait plusieurs provinces. ${ }^{24}$

La pâtisserie de la Grand'rue: en toile de fond on retrouve bien évidemment Chaminadour, dont l'auteur parlera ailleurs comme de « la capitale de son imagination $»^{25}$ - Mais cette province, où commence le "long exercice de déchirement $»^{26}$, étape fondamentale de la formation de Jouhandeau, est un lieu habité par des monstres ${ }^{27}$. En effet, on découvre au paragraphe suivant qu'une des Malinjoude, Alexandrine, s'était laissé séduire par on ne sait qui au cours d'un long voyage : elle avait accouché ensuite «d'un garçon chevelu tout armé d'ongles et de dents $»^{28}$ qu'elle avait voulu appeler Ximénès, « on eut beau lui demander pourquoi $»^{29}$, commente le narrateur, la femme n'en donnait pas l'explication. Ximénès est une variante de Jiménez : l'étymologie est basque et le nom signifie «fils"; Jouhandeau ne l'ignorait probablement pas, ayant insisté à plusieurs reprises sur l'attention qu'il portait au choix des noms de ses personnages ${ }^{30}$.

Ximénès, dont le corps est enveloppé d'une certaine "phosphorescence fugitive " ${ }^{31}$, est le fils du Diable; il se définit comme l'Assassin de Dieu, menant « un corps à corps avec l'Éternel $\aleph^{32}$. Au cours de la narration, il accomplit plusieurs meurtres gratuits ${ }^{33}$ jusqu'à l'apparition du personnage d'Amélin, un garçon "à la bouche d'Archange ॥ $^{34}$ représentant pour Ximénès sa chance d'échapper à la damnation : les échanges entre eux deux ne sont rien moins qu'un combat mythologique entre le Bien et le Mal. Dans les dernières scènes, leur dialogue atteint une densité paroxysmique et les lectures possibles se multiplient. Ximénès demande à Amélin de l'aider à tuer Dieu, puis de lui ressembler; ce leitmotiv de la ressemblance physique est tout à fait diabolique : c'est en effet le Diable qui veut qu'on lui ressemble.

19 Le thème de la ressemblance physique est récurrent chez Jouhandeau : ses personnages sont souvent obsédés par une même image qui revient, de même que l'auteur idolâtrait 
une beauté masculine idéale, comme on le comprend notamment dans les écrits érotiques publiés sous forme anonyme à partir de la fin des années 1940. Dans Tirésias, par exemple, c'est Pierre, le dernier des amants du narrateur, qui apparaît et disparaît comme un démon ; à la fin du récit, la toute dernière phrase est une allusion explicite au fait qu'il pourrait être le Diable ${ }^{35}$. Ce fil qui relie le Diable à Dieu et à l'homme est fondamental dans la construction de l'éthique de Jouhandeau, qui définit une existence à la fois libertine et mystique. La ressemblance, la proximité entre le Diable, Dieu et l'homme est la clé de son "algèbre métaphysique " ${ }^{36}:$ si l'homme est fait à l'image de Dieu, aimer l'homme est théologiquement permis, voire souhaité. Au cours de la narration, Brice Aubusson - le protagoniste du roman L'Amateur d'imprudence, le deuxième dont je traiterai ici - dessine trois fois le croquis de ce visage qui l'a «tenté »; et dit de ce personnage qui incarne la Tentation qu'il lui ressemble.

Le plan du combat entre le Ciel et l'Enfer se superpose ainsi au plan de l'appel continu du désir homosexuel. Dans Ximénès Malinjoude, le caractère érotique de la relation entre le protagoniste et Amélin est palpable, leur attrait réciproque est explicite. Et, de surcroit, l'auteur fait dire à Ximénès qui s'adresse à Amélin :

Sais-tu [...] quelle seule différence entre toi et moi subsiste ? [...] C'est que j'ai le courage de mes désirs et que tu as peur des tiens, mais je t'amènerai bientôt peu à peu à connaître l'invincible Pureté qui est la mienne, à ne plus avoir de remords, à désirer de me ressembler tout à fait. ${ }^{37}$

21 Ce chemin de la perdition tracé par Ximénès est aussi le chemin de ce que Jouhandeau appelle le Vice, la passion de la chair, la tentation homosexuelle. Cependant, il le répétera à plusieurs reprises, il n'y a pas de vertu sans vice. Écrit dans une prose riche et élégante, Ximénès Malinjoude est un prodigieux récit cruel illustrant à merveille «le passage de la littérature française du symbolisme au surréalisme $»^{38}$. Publié en 1927 puis en 1930, mais pensé déjà en 1924, on pourrait y voir un point de départ à cette autobiographie d'un "pécheur habitué ${ }^{39}$ que constitue l'œuvre de Jouhandeau: au beau milieu de cette province monstrueuse où tout commence, l'auteur met en scène un combat entre le Bien et le Mal. À la fin, le Bien tue le Mal pour s'en libérer : mais le meurtre n'est-il pas déjà le Mal ? La lutte avec les démons est une constante de l'œuvre de Jouhandeau : c'est une lutte sans fin, ces démons se reproduisant en série puisqu'ils représentent chacun une tentative échouée d'incarner la forme divine.

La relation ambiguë existant entre le Bien et le Mal est une constante dans la production de Jouhandeau: à travers ses livres, à partir de Chaminadour, l'auteur élabore « une étrange théologie sans fin tissée autour de quatre pôles : le Bien et le Mal, l'Être, l'Éternel et le Singulier $\aleph^{40}$. On voit clairement que le Bien et le Mal constituent un même pôle. Au sein de cette théologie, le Ciel est Dieu, ou l'Éternité, alors que l'Enfer est la passion, la recherche chez l'Homme de cette forme divine qui fait que l'éthique de Jouhandeau est d'abord une esthétique ${ }^{41}$.

\section{L'Amateur d'imprudence. Variations sur le Bien et le Mal}

23 Ce climat d'ambiguïté, d'hésitation perpétuelle entre le Bien et le Mal, s'impose également dans L'Amateur d'imprudence, roman publié en 1932. Le protagoniste, Brice Aubusson, est obsédé par la recherche de l'Idéal et hanté par le visage d'un personnage qui à nouveau apparaît et disparaît comme un démon; on découvre peu à peu qui est vraiment ce personnage au cours de la narration. Il est intéressant à cet égard de relever 
que ce dernier est introduit dans le roman par le pronom impersonnel « on ». Lors de la fête qui tient lieu de toile de fond à la première scène importante de L'Amateur, Pia, qui accompagne Brice, veut que son ami lui désigne l'être qui l'obsède : « On est là ? ${ }^{42}$ est la question qu'elle lui pose sans cesse. Bien que le pronom impersonnel serve à l'auteur à ne pas préciser le genre de l'objet désiré, que Pia croit d'abord être une femme, on peut constater que cet usage contribue à dépersonnaliser l'obsession de Brice et à lui accorder plus de mystère.

Comme on l'a vu précédemment, les hommes aimés et racontés par Jouhandeau ont souvent quelque chose de fantasmatique: si Ximénès était le fils du Diable, la figure infernale par excellence façonne plusieurs êtres nés du génie jouhandélien, les rendant au moins partiellement démoniaques. L'homme désiré par l'auteur - irréel, mythique, image incarnée du péché - relève du domaine du fabuleux. Dans une des scènes les plus fantasques de L'Amateur, Brice finit par se retrouver dans une sorte de laboratoire de l'Idéal, où l'on peut composer le corps de l'amant parfait, en choisissant d'abord les bras les plus beaux dans la chambre des bras, puis les jambes les plus musclées dans la salle des jambes, et ainsi de suite.

L'atmosphère onirique de L'Amateur d'imprudence est superbement maîtrisée par l'auteur, le rêve alternant avec l'imagerie, voire avec des sortes d'ekphrasis hallucinées. Escorté par Lipse, personnification de l'imprudence et du danger ${ }^{43}$, Brice entre dans une dimension magique : les deux plongent dans les fresques des églises de San Pietro Martire ou de Sant'Anastasia à Vérone, puis Brice se retrouve dans un lit avec deux cadavres à ses côtés: il s'agit de Roméo et Juliette, qui incarnent la Beauté idéale. À cette série d'apparitions, à ce registre visionnaire s'entremêlent les commentaires du narrateur ou $\mathrm{du}$ protagoniste, parfois exprimés dans une prose elliptique, alambiquée, voire vertigineuse, qui aura peut-être découragé certains lecteurs. En voici un exemple (c'est Brice qui parle) :

Le plus terrible, c'est que je souffre et que je crois jouer la comédie, que je crois que ma douleur n'est qu'une comédie, que ma douleur n'est peut-être qu'une comédie encore quand je souffre et que je souffre comme si je souffrais et encore par dessus le marché que ma douleur ne soit qu'une comédie, si bien que je ne sais plus à la fin si je souffre ou si je feins de souffrir et que je souffre de feindre, mais que je finis purement et simplement tantôt par feindre sans mélange et tantôt par souffrir et que ma feinte et ma souffrance ne se mêlent pas et que de feindre m'est une joie si je ne souffre plus, que de souffrir m'est une joie si je ne feins plus et que je finis purement et simplement par me réjouir et de ma feinte et de ma souffrance. C'est dire qu'au moment où je souffre, je voudrais bien ne plus souffrir et alors je me souviens que je feins et à cette idée que je feins, j'éprouve une consolation infinie : je ne souffre plus, je feins, je m'en réjouis. Mais dès que je m'aperçois que je feins, mon cœur déplore l'artifice : il faut que je souffre. Je souffre et de souffrir encore m'est une joie, s'il me console de feindre. ${ }^{44}$

Cet extrait représente un cas exceptionnel de syntaxe hypertrophique, mais il montre néanmoins la virtuosité stylistique que Jouhandeau possédait et utilisait à sa guise. Il en joue ailleurs avec encore plus d'efficacité, par exemple dans ce portrait de Brice, esquissé dans une prose savamment agencée :

S'il est vrai que la personnalité s'inscrit dans le schème des figures que décrit le filigrane de nos gestes sur celui de nos regards et inversement celui de nos regards sur celui de nos gestes et qu'on pourrait mesurer l'élévation morale d'un être à la distance qu'il sait maintenir entre ses yeux et ses mains, sa délicatesse à la subtilité qu'il entretient dans leurs rapports continuels, quel n'était pas Brice pour qu'un 
tissu de lignes aussi indélébiles, il est vrai, qu'invisibles, tracées dans l'espace, fût si

singulier qu'il suffit à déceler sa présence, à l'identifier. ${ }^{45}$ n'était pas censé s'intéresser à un roman qui représente « un exemple parfait de poème surréaliste $»^{46}$, et où c'est précisément à travers des visions déséquilibrées que l'auteur parvient à percer le secret de ses personnages. Cependant, c'est dans L'Amateur que Jouhandeau, sous les traits d'un nouvel alter ego créé après Monsieur Godeau, définit clairement son cadre moral et existentiel. «La vertu n'a pas d'autre étoffe que le vice de notre désir qu'elle corrige et dirige ${ }^{47}$, affirme le protagoniste ; ou encore :

Je ne comprends pas le vice en effet, s'il est sans rapport avec la vertu ni la vertu, si elle est étrangère au vice. [...] Le Drame seul m'intéresse que créent le Mal et le Bien. Mais je n'ai de préférence ni pour le Bien ni pour le Mal, si je n'aime le Courage. ${ }^{48}$

rès la « religion du mal » professée par Ximénès, dans L’Amateur d'imprudence le Bien et le Mal deviennent interchangeables. On commence alors à identifier les paradigmes jouhandéliens : la Vertu est l'imprudence ; l'imprudence est le contraire de la médiocrité, le danger du Vice ; et le Vice est « le désir à l'état pur » ${ }^{49}$, lit-on dans Éloge de l'imprudence, un court essai publié avec le roman. L'auteur n'utilise que des termes relevant du domaine de l'Absolu, car c'est bien l'Absolu qui constitue le cadre de la quête de soi : Brice Aubusson est Monsieur Godeau sans masque, qui a compris que «sa démarche dans le péché n'est pas différente de celle du Saint " $^{50}$. Comme Jouhandeau, Brice est « délivré de tout, du Temps, de l'Espace $»^{51}$, et cela grâce à un ange nécessaire, énième incarnation d'un désir aux mille visages, traversant l'ensemble de l'œuvre de Jouhandeau, dans ce combat éternel entre le Ciel et l'Enfer.

Un tel combat détourne de l'œuvre de l'auteur à la fois les croyants, qui ne comprennent ni ne peuvent comprendre son mysticisme hérétique, et certains non-croyants, pour qui ses acrobaties de moraliste semblent insolites et un peu déplacées sur une scène littéraire de plus en plus dominée par la littérature engagée.

\section{Le prix de la singularité et de l'éternité}

Comme le dit Henri Mitterand, la question du Mal, pris dans son acception politique, a envahi la conscience des romanciers depuis 1930 :

À gauche, le Mal, c'est le capitalisme, la dictature, l'ordre bourgeois, la corruption par l'argent, le carriérisme, la dictature, les généraux rebelles, l'exploitation de l'homme par l'homme, l'approche d'une guerre qu'on devine plus horrible que la précédente; à droite, c'est la démocratie, le parlementarisme, la veulerie des masses, etc. ${ }^{52}$

Pour Mitterand, Jouhandeau fait partie de ces auteurs qui entendent rester à l'écart des luttes sociales et politiques et pour qui le Mal est organique à l'homme. La recherche jouhandélienne sur le mal s'inscrit ainsi dans le cadre de l'absolu, dépassant les frontières historiques de son époque : l'éternité est le seul « registre » de l'auteur, intéressé par son « ipséité éternelle », pour reprendre l'expression de Bounoure citée au début.

Dans son oeuvre, Jouhandeau aborde à plusieurs reprises la question identitaire, notamment dans ses nombreux Journaliers :

On renonce le plus souvent à être soi, par conformisme ou paresse, parce que rien n'est plus difficile que de s'insérer dans le monde, quand on ne lui ressemble pas, 
quand on porte en soi quelque chose d'imprévu, d'incomparable, d'extraordinaire (et je ne mets dans ces trois épithètes aucun orgueil)..$^{53}$ car c'est bien l'ensemble de sa production qui donne son sens à cette autobiographie par étapes, intéressante en cela qu'elle dit l'évolution progressive d'un homme ayant toujours cherché à se comprendre en profondeur et à dépasser ses limites. À travers ses écrits, Jouhandeau a tenté la synthèse de l'Érotisme et de la Théologie, du désir sensuel et de l'appel mystique. Ximénès, Brice, Monsieur Godeau et les autres évoluent à travers son œuvre "comme des marionnettes emplies d'un souffle surnaturel $»^{56}$. La touche d'éternité déjà soulignée ici est liée notamment à ce que Gaulmier appelle l'essentialisme de ses personnages. Gaulmier entend par ce terme la voie opposée à l'existentialisme : alors que les actes des personnages sartriens et des autres existentialistes se présentaient comme la résultante de situations, ils sont chez Jouhandeau le produit d'une activité volontaire sans rapport avec le contexte. Il s'agit de personnages hors du temps et de l'espace, d'âmes singulières dont la psychologie se rattache à un archétype, «à une essence éternelle $»^{57}$.

Cet aspect constitue une raison de plus pour classer Jouhandeau parmi les auteurs inclassables, ceux qui semblent avoir écrit pour eux-mêmes plus que pour leur public. Ainsi, pour entrer en contact avec le monde jouhandélien, il est nécessaire de rechercher une complicité avec l'auteur. Cette complicité commence par la compréhension de sa religion du péché parfait, véritable clef de lecture de l'ensemble de son œuvre: dans sa province plus ou moins monstrueuse, Jouhandeau ouvre son parcours de recherche sur le bien et le mal pour finalement n'exclure de son univers aucun des deux; dans Ximénès Malinjoude notamment (qui fut classé dans les Contes d'enfer bien qu'ayant Chaminadour comme toile de fond), l'auteur se libère de la haine de Dieu et commence en quelque sorte à mettre Dieu et l'homme sur le même plan : c'est le point de départ de sa théologie du désir. Plus tard, il considère que le plaisir n'a pas la même saveur sans l'imprudence (c'est ce que l'on avait vu dans L'Amateur d'imprudence); il réintègre alors la notion de péché à l'intérieur de son monde, notamment grâce à l'expédient narratif du mariage, évoqué dans plusieurs œuvres (Monsieur Godeau marié, Chroniques maritales, etc.) ${ }^{58}$. 
Tout cela n'est qu'une partie de cet étrange ballet d'un pécheur moraliste que fut la vie de Jouhandeau. Héron dit que cette vie s'est déroulée en quatre étapes: de l'expiation à l'abjection, de l'abjection à la vie, de la vie à la fête, de la fête à l'espérance ${ }^{59}$. Je dirais que les deux œuvres ici traitées, ainsi que la production jouhandélienne des années 30, appartiennent plutôt à la deuxième phase, une phase capitale : de l'abjection à la vie.

\section{Conclusion}

En 1959, on commençait tout juste à considérer Jouhandeau comme l'un des maîtres de la littérature française ${ }^{60}:$ de toute évidence, cette renommée n'a guère duré. Avec son culte obsessionnel de la singularité, ses élans mystiques et son désir inépuisable d'atteindre l'éternel, il parlait sans doute une langue que de moins en moins de lecteurs souhaitaient entendre. À cela s'ajoute son désintérêt pour la réalité qui l'entourait, maintes fois confirmé, face à une scène littéraire de plus en plus engagée : il ne s'est en effet jamais senti concerné par la politique, car tout ce qui comptait pour lui, c'était l'Homme, unique et infini.

Ma vocation repose toute sur l'estime singulière que j'ai conçue pour mon âme et pour l'âme humaine, dès l'origine de ma vie. Dès mon enfance, le sentiment de ma propre grandeur m'a accablé et le sentiment de la grandeur de n'importe qui m'a troublé, enivré pour toujours, comblé. ${ }^{61}$

Jouhandeau a mis l'âme humaine au cœur d'une recherche qui a duré toute une vie : ses récits, contes, nouvelles, anecdotes, portraits, ainsi que ses nombreux «journaliers », en sont un époustouflant témoignage; ils composent le récit d'une vie marquée par un intense désir de sonder la trame de l'existence, qui peut captiver l'intérêt du lecteur d'aujourd'hui tout comme il le captivait à son époque. Si l'on peut affirmer qu'aucun véritable chef-d'œuvre ne se démarque de l'ensemble de l'œuvre de Jouhandeau, c'est précisément dans cette constellation fragmentée que l'on retrouve une autobiographie permanente, mi-romancée, dans cet ensemble qui

constitue une sorte de journal où Jouhandeau relate son expérience de soi et d'autrui, prend la mesure ineffable de son âme, une série d'essais plutôt que de romans, en donnant à ce mot d'essai son sens plein de tentatives multipliées en vue de serrer de plus près une réalité psychologique toujours fuyante. ${ }^{62}$

Chez Jouhandeau, chacune des œuvres n'acquiert pleinement sa valeur qu'en lien avec les autres. Livre après livre, cet auteur, narrateur protéiforme, bâtit un monde « tout neuf, bien à [lui], à triple atmosphère $»^{63}$, qui mérite d'être redécouvert.

41 «Lire Jouhandeau - a écrit Richard Millet - c'est entrer dans un chemin d'orties et de lys; c'est aller de l'enfer au ciel et inversement ${ }^{64}$. Lire Jouhandeau, c'est enfin partager, dans une prose magnifique et une singularité totale, la multiplicité foisonnante des actes et des rêves d'un auteur qui aurait pu sans aucun doute dire avec Térence « Homo sum : humani nihil a me alienum puto ». 


\section{NOTES}

1. M. Jouhandeau, Correspondance avec André Gide. Octobre 1922-Septembre 1946, Paris, Sautier, 1958, p. 29.

2. Ces deux périphrases appartiennent à des détracteurs de Jouhandeau. La première est d'André Rousseaux, Littérature du vingtième siècle, t. IV, Paris, Albin Michel, 1953, p. 219. La seconde paraît sur L'Osservatore Romano, quotidien d'information du Vatican, en 1953. Jouhandeau répondra à l'article en 1963, dans La Revue de Paris. La reconstruction de cet échange, avec article et réponse, se trouve en appendice à M. Jouhandeau, Tiresia, éd. O. Tajani, Napoli, Marchese, 2013 (avec un essai de Giuseppe Merlino).

3. La définition est utilisée par Gabriel Bounoure à propos du personnage de Monsieur Godeau, sous le masque duquel l'auteur aimait à se cacher. Cf. G. Bounoure, "Notes sur Marcel Jouhandeau ", dans La Nouvelle Revue Française, 158, novembre 1926, p. 626-632.

4. M. Jouhandeau, Essai sur moi-même, Paris, Gallimard, 1947, p. 69.

5. Ibid., p. 66.

6. Ibid., p. 65.

7. M. Jouhandeau, Algèbre des valeurs morales, Paris, Gallimard, 1935, p. 176.

8. M. Jouhandeau, Éléments pour une éthique, Ermenonville, Noé, 2006, p. 36.

9. B. Meyer, Éros jouhandélien: l'attrait de l'homme dans les premières cuvres de Marcel Jouhandeau, Paris, L'Harmattan, «Saint-Denis-de-La-Réunion, CRLH-Université de La Réunion », 1994, p. 104-105.

10. M. Jouhandeau, De l'Abjection, Paris, Gallimard, 1939, p. 48.

11. J.-P. Sartre, «Compte rendu de La Possession», dans Bulletin bibliographique et critique, 295, juillet 1970, p. 679.

12. R. Muchembled, Une histoire du Diable. XII ${ }^{e}-X X^{e}$ siècle, Paris, Seuil, 2000, p. 286, 290.

13. J. Gaulmier, L'Univers de Marcel Jouhandeau, Paris, Nizet, 1959, p 145.

14. R. Millet, "Préface», dans M. Jouhandeau, Chaminadour: contes, nouvelles et récits, Paris, Gallimard, « Quarto », 2006, p. 16.

15. C. Mauriac, Introduction à une mystique de l'Enfer. L'œuvre de Marcel Jouhandeau, Paris, Grasset, 1938, p. 22-26. Bien qu'animé par la velléité de faire de Jouhandeau un écrivain catholique, le livre de Mauriac, riche en intuitions, est le premier ouvrage important consacré à l'auteur.

16. On trouve une analyse intéressante de la question de l'antisémitisme chez Jouhandeau dans l'ouvrage de D. Eribon, Hérésies : essais sur la théorie de la sexualité, Paris, Fayard, 2003, p. 175-188.

17. Trois crimes rituels (Tre delitti rituali, Milano, Adelphi, 1996); Chroniques maritales (Cronache maritali, Milano, Adelphi, 1999) ; Tirésias (Tiresia, Napoli, Marchese, 2013).

18. J. Gaulmier, op. cit., p. 33.

19. M. Jouhandeau, Essai sur moi-même, cit., p. 65.

20. À ce sujet, cf. aussi B. Meyer, Éros jouhandélien : l'attrait de l'homme dans les premières œuvres de Marcel Jouhandeau, cit. Meyer focalise son attention sur les œuvres antérieures à 1931, son but étant de reconnaître chez l'auteur les traces d'une homosexualité encore cachée.

21. Cf. M. Leiris, Journal 1922-1989, Paris, Gallimard, 1992, pp. 47, 49 etc. pour des allusions à leur «aventure ». P.-M. Héron en parle aussi dans Marcel Jouhandeau. L'orgueil de l'homme, Limoges, Pulim, 2009, p. 33.

22. M. Jouhandeau, Ximénès Malinjoude, dans Chaminadour, cit., p. 530.

23. H. Rode, "Compte rendu de Contes d'enfer de Marcel Jouhandeau», dans Cahiers du Sud, t. XLIII, n. 337, octobre 1956, pp. 446-447. 
24. M. Jouhandeau, Ximénès Malinjoude, dans Chaminadour, cit., p. 519.

25. M. Jouhandeau, L'arbre de visages dans Chaminadour, cit., p. 1288.

26. M. Jouhandeau, Éléments pour une éthique, cit., p. 12 (préface de Jacques Rougeon).

27. Cf. aussi P.-M. Héron, op. cit., p. 18.

28. M. Jouhandeau, Ximénès Malinjoude, cit., p. 519.

29. Ibidem

30. Gaulmier lui aussi le relève, cf. J. Gaulmier, op. cit., p. 127-128.

31. M. Jouhandeau, Ximénès Malinjoude, cit., p. 538.

32. Ibid., p. 543.

33. Le meurtre gratuit suscite l'intérêt de Jouhandeau pour l'analyse de l'«inhumanité satanique faite homme ", selon les mots de Pierre-Marie Héron (cf. P.-M. Héron, op. cit., p. 42) ; intérêt dont l'auteur témoignera également lors de l'écriture de Trois crimes rituels, recueil des récits de trois crimes sordides des années 1950 dont Jouhandeau suivit les procès. Ce genre d'«acte gratuit " s'inscrit pour l'auteur dans une dimension existentielle mythique, dans un registre hautement symbolique et a donc peu à voir, par exemple, avec l'acte du Lafcadio gidien dans Les Caves du Vatican. Rappelons que pour Yvonne Davet, ce dernier livre est « résolument satirique et parodique", et l'acte accompli par Lafcadio n'est que la caricature d'un acte « hautement significatif » (voir A. Gide, Romans, récits et soties, éd. Y. Davet et J.-J. Thierry, Paris, Gallimard, «Bibliothèque de la Pléiade », 1958, p. 1571).

Jouhandeau et Gide étaient liés d'amitié, et il est facile de reconnaître chez eux un intérêt commun pour un certain nombre de sujets (l'homosexualité, le mariage, la vie morale, etc.). Cependant, leurs tempéraments littéraires étaient très différents. Ce n'est pas ici le lieu pour approfondir la question, et l'on se contentera pour l'heure de rappeler ce que disait Blanchot de notre auteur dans Faux pas, qui l'éloigne diamétralement de Gide : « Tout l'art de Jouhandeau est de rendre pathétique la montée au symbole». Alors qu'un des traits les plus manifestement gidiens était l'ironie, le «symbolisme pathétique » jouhandélien n'était guère ironique, et fut tout au plus, à l'occasion, tempéré par l'auto-ironie. Et si Gide affichait une tendance désacralisante, Jouhandeau tendait plutôt à sacraliser tout ce qu'il touchait.

34. M. Jouhandeau, Ximénès Malinjoude, cit., p. 544.

35. M. Jouhandeau, Tirésias, Paris, Arléa, 1988, p. 92.

36. B. Meyer, op. cit., p. 85.

37. M. Jouhandeau, Ximénès Malinjoude, cit., p. 544.

38. J. Gaulmier, op. cit., p. 149.

39. L'expression est tirée de M. Jouhandeau, Monsieur Godeau intime, Paris, Gallimard, 1982, p. 46.

40. B. Sichère, Le dieu des écrivains, Paris, Gallimard, 1999, p. 110.

41. Claude Mauriac le note aussi dans son ouvrage, cf. C. Mauriac, op. cit., p. 165.

42. M. Jouhandeau, L'Amateur d'imprudence, Paris, Gallimard, 1932, p. 23.

43. C'est Brice lui-même qui le dit, cf. ibid., p. 186.

44. Ibid., p. 31-32.

45. M. Jouhandeau, L'Amateur d'imprudence, cit., p. 33.

46. J. Gaulmier, op. cit., p. 53.

47. M. Jouhandeau, L'Amateur d'imprudence, cit., p. 14-15.

48. Ibid., p. 42.

49. M. Jouhandeau, Éloge de l'imprudence, Ermenonville, Noé, 2006, p. 36-37.

50. H. Rode, Marcel Jouhandeau et ses personnages, Paris, Éditions de la Tête de feuilles, 1972, p. 43.

51. M. Jouhandeau, L'Amateur d'imprudence, cit., p. 49.

52. H. Mitterand, La littérature française du XXe siècle, Paris, Colin, 2007, p. 35.

53. M. Jouhandeau, Être inimitable. L'Hermitage. Adieu à la Porte Maillot (Journaliers VI), Paris, Gallimard, 1964, p. 21. 
54. M. Jouhandeau, Dans l'épouvante, le sourire aux lèvres. Décembre 1973 - Noël 1979 (Journaliers XXVIII), Paris, Gallimard, 1982, p. 190.

55. M. Blanchot, Faux pas, Paris, Gallimard, 1971, p. 261.

56. Critique de G. Bounoure, op. cit., citée aussi dans J. Gaulmier, op. cit., 48.

57. Ibid., p. 37.

58. On pourrait être tenté d'affirmer que Jouhandeau ne se maria que pour pouvoir écrire sur les douleurs de son mariage, pour continuer à raconter son cheminement dans ce qu'il appelle le péché, ajoutant la faute morale à l'impiété. Ce serait peut-être excessif, mais il est vrai que l'écriture suit la biographie de l'auteur en recréant une ambiguïté qu'on aura désormais appris à connaître : si, comme d'autres l'ont déjà remarqué, chez lui « la passion et l'écriture de la passion se ressemblent au point de former comme une même activité onirique » (M. Jouhandeau, Éloge de l'imprudence, cit., p. 21), aux étapes de sa production littéraire correspond une évolution humaine et existentielle qui ne cesse de susciter l'intérêt du lecteur.

59. P.-M. Héron, op. cit., p. 167.

60. J. Gaulmier, op. cit., p. 5.

61. M. Jouhandeau, Essai sur moi-même, cit., p. 66.

62. J. Gaulmier, op. cit., p. 11.

63. M. Jouhandeau, Essai sur moi-même, cit., p. 65.

64. R. Millet, « Préface », dans M. Jouhandeau, Chaminadour, cit., p. 17.

\section{RÉSUMÉS}

Une figure de l'enfer dont l'œuvre est tombée dans le purgatoire de l'oubli : la fortune éditoriale de Marcel Jouhandeau est difficile à saisir. Ses collègues de l'époque, dont André Gide, admirent sa prose ; la critique lui reconnaît un talent hors du commun; les éditions Gallimard publient presque tous ses écrits. Cependant, aujourd'hui, on le connaît moins que ses cent-vingt titres publiés ne le feraient imaginer. À cet oubli, maintes raisons possibles. Dans cet article, je donnerai une vue d'ensemble de ces raisons avant d'introduire l'idée que l'œuvre jouhandélienne constitue une sorte de grande autobiographie fragmentée : à chaque volume publié correspond une étape de la formation humaine de l'auteur. En m'appuyant sur des références aux œuvres Ximénès Malinjoude et L'Amateur d'imprudence notamment, je montrerai qu'au milieu de l'univers de Jouhandeau, il y a la recherche de la vérité de l'Homme. Ce n'est que sous cette lumière que sa production acquiert tout son sens ; ce n'est que grâce à cette clé que le lecteur peut plonger dans l'œuvre de cet auteur et l'apprécier pleinement encore aujourd'hui.

\section{INDEX}

Mots-clés : Oubliés, Jouhandeau (Marcel), autobiographie, Ximénès Malinjoude, L'Amateur d'imprudence, années Trente 